\title{
Evolving spiking neural networks methods for classification problem: a case study in flood events risk assessment
}

\author{
Mohd Hafizul Afifi Abdullah', Muhaini Othman', Shahreen Kasim ${ }^{3}$, Siti Aisyah Mohamed \\ ${ }^{1,2,3,4}$ Faculty of Computer Science and Information Technology, Universiti Tun Husssein Onn Malaysia, Malaysia \\ ${ }^{1,3}$ Soft-Computing and Data Mining Center, Universiti Tun Husssein Onn Malaysia, Malaysia
}

\begin{tabular}{l} 
Article Info \\
\hline Article history: \\
Received Jan 21, 2019 \\
Revised Mar 26, 2019 \\
Accepted Apr 15, 2019 \\
\hline
\end{tabular}

\section{Keywords:}

Classification

Data mining

Pattern recognition

Personalised modelling

Spiking neural networks

\begin{abstract}
Analysing environmental events such as predicting the risk of flood is considered as a challenging task due to the dynamic behaviour of the data. One way to correctly predict the risk of such events is by gathering as much of related historical data and analyse the correlation between the features which contribute to the event occurrences. Inspired by the brain working mechanism, the spiking neural networks have proven the capability of revealing a significant association between different variables spike behaviour during an event. Personalised modelling, on the other hand, allows a personal model to be created for a specific data model and experiment. Therefore, a personalised modelling method incorporating spiking neural network is used to create a personalised model for assessing a real-world flood case study in Kuala Krai, Kelantan based on historical data of 2012-2016 provided by Malaysian Meteorological Department. The result shows that the method produces the highest accuracy among the selected compared algorithms.
\end{abstract}

Copyright @ 2019 Institute of Advanced Engineering and Science. All rights reserved.

\section{Corresponding Author:}

Muhaini Othman,

Faculty of Computer Science and Information Technology,

Universiti Tun Hussein Onn Malaysia,

86400 Parit Raja, Batu Pahat, Johor, Malaysia.

Email: muhaini@uthm.edu.my

\section{INTRODUCTION}

In a data-driven world we live in today, technology advancement has made it possible to process vast amount of data for analysing pattern of events occurrences. These data is used in different localities, for instance, in constructing a predictive data model for weather and environmental events forecasting, traffic analysis, sentiment analysis, and many other applications. Development of hardware and software for datadriven technology is limitless. An example of applied data-driven technology has been published in Kasabov et al. [1] where data analysis has been applied on ecological data, health-related data, aphid population prediction, and others. Data-driven technology development and application has also been applied on a neuromorphic hardware [1] to better-visualise the constructed predictive models.

Kasabov [2, 3] has addressed that most environment-related event can be captured to form spatio/spectro-temporal data (SSTD) by capturing data from different places (spatio-component or localities) and their corresponding variables timely measurements. Several works have also been published on ecological data $[4,5]$, stroke data $[1,4,6-9]$, and learning of spatio-temporal brain data $[10,11]$, demonstrating the capability of a computational predictive method for extraction of knowledge from SSTD.

Analysing and understanding SSTD is considered as a challenging task due to the close interaction and interrelationship between the spatial/spectral-components with the temporal-components [4], the nonsuitability of a conventional machine learning to be used for classification of SSTD (only suitable for classifying vector-based and static type of data) [10], and the training samples and testing samples must have 
the same number of input features-hence eliminating some input to produce consistent number of features causes information loss [4].

An investigation on natural events requires analysis on complex high dimensional data in the form of spatio- and spectro-temporal data (SSTD) and it is important to retain the interrelationship information between the components. Data mining tools provides a promising framework for discovering hidden patterns within a multi-dimensional dataset, allowing prediction of occurrences-applicable in various domains like prediction of environmental disaster occurrences, risk of stroke [1, 3, 4, 6-9], assessment of ecological data [5, 12, 13] prediction of unknown gene functions [14], and many others [7, 15-17].

In ecological and river engineering aspects, Ghani et al. $[18,19]$ have summarised that flood is caused by meteorological factors (climate, as well as duration and intensity of rainfall), geological features, and urbanisation process. Sinnakaudan et al. [20] have stated that floods, apart of being caused by the random coincidence of several meteorological factors by nature-the severity and consequences of the events are also influenced by man's use of the river's catchment. Theoretically, it is possible to accurately predict environmental events such as flood cases by analysing SSTD present in the environmental historical data.

Therefore, this paper presents an approach for solving classification problems, adaptively applied for assessment of flood risks by using evolving Spiking Neural Networks methods. The subject involves 5 years environmental-related temporal data (from 2012 until 2016) of Kuala Krai in Kelantan, Malaysia. This article assumes less prior knowledge on data modelling techniques and neural network generations.

\section{Data Modelling Techniques}

Global Modelling — defined by a single function for the whole problem space [2, 21], global modelling is applied in algorithms such as in Support Vector Machine (SVM) and Multi-Layer Perceptron (MLP). SVM consists of a kernel function, basically an equation which divides data vectors into different classes based on which area the data vector falls on $[2,21]$. A model created using global modelling can be easily applied to new data, however, additional knowledge regarding the data such as the nature of the data and knowledgedatabase is naturally neglected in global modelling which causes information loss [3, 12]. Due to this characteristic of global modelling, it is not suitable to be used to analyse the dynamic and

Local Modelling — introduced to solve problems of global modelling, where local modelling is more adaptable to new data vectors [2, 21]. According to Kasabov [3], local modelling is introduced by forming subsets of the whole problem space, hence a whole problem space is represented by multiple subsets called classes. Local modelling algorithms include $K$-means, Self-Organizing Maps (SOM), Fuzzy Clustering, and Hierarchical Clustering

Personalised Modelling — created based on a single point from a subset of the whole problem space, in which every new data vectors can be classified into their corresponding classes based on the model which is constructed "on the fly" $[3,12,22] . K$-Nearest Neighbor $(K-\mathrm{NN})$ is a modelling technique which for every new samples, the nearest $K$ samples are derived from the data set using Euclidean distance measure and a personalized voting which then labels the samples to its appropriate cluster [3].

Table 1 summarizes the comparison between global, local, and personalised modelling techniques which highlights on the important criteria such as problem set covered, benefits, and limitations.

Table 1. Comparison between global, local and personalised modelling techniques

\begin{tabular}{|c|c|c|c|}
\hline Techniques & Global Modelling & Local Modelling & Personalised Modelling \\
\hline Problem Set & Entire problem space & $\begin{array}{l}\text { A cluster from the entire } \\
\text { problem space }\end{array}$ & $\begin{array}{l}\text { A single point from the a cluster in } \\
\text { the entire problem space }\end{array}$ \\
\hline Reasoning Theory & Inductive reasoning & Transductive reasoning & Transductive reasoning \\
\hline Applicable & SVM & $K$-means, SOM, Fuzzy & $K$-NN, W $K$-NN, WW $K$-NN \\
\hline Algorithms & & $\begin{array}{l}\text { Clustering, Hierarchical } \\
\text { Clustering }\end{array}$ & \\
\hline Benefits & $\begin{array}{l}\text { Provide overview (big } \\
\text { picture) of the knowledge } \\
\text { without knowing the } \\
\text { details }\end{array}$ & $\begin{array}{l}\text { Easier to adapt new data and } \\
\text { e provides better explanation } \\
\text { for individual cases }\end{array}$ & $\begin{array}{l}\text { New input data can be labeled to } \\
\text { its corresponding cluster on the fly }\end{array}$ \\
\hline Limitations & $\begin{array}{l}\text { Offers limited knowledge } \\
\text { to be extracted from the } \\
\text { output data; difficult to } \\
\text { adapt to new data }\end{array}$ & $\begin{array}{l}\text { Requires knowledge on } \\
\text { number of available clusters } \\
\text { via input or cluster } \\
\text { initialization; thus cannot } \\
\text { construct model on the fly }\end{array}$ & $\begin{array}{l}\text { Employs much more complex } \\
\text { algorithm than global and local } \\
\text { modelling }\end{array}$ \\
\hline
\end{tabular}

\section{Neural Networks Generations}

The First Generation of Neural Networks. Considering computational units are used to define the generations of neural networks, the networks employing the McCulloh-Pitts neurons (known as perceptrons) 
is the first generation of neural networks. The neuron body is able to make calculations based on multiple inputs, producing a single output as such inputs are $x$ and $y$, neuron body processes $z=x+y$, and output is $z$. It is capable to solve simplex problems, but does not to provide conditioning statements to the produced output.

The Second Generation of Neural Networks. The idea behind this is to integrate an activation function. Each neuron receives multiple inputs, processed by the neuron function, and as the output value from the neuron surpasses the activation function threshold, an output will be produced. Various activation function can be integerated into the neuron includes sigmoid, Rectified Linear Unit (ReLU), and linear activation functions.

The Third Generation of Neural Networks. Spiking neural network (SNN), employing spiking neurons (also known as integrate-and-fire neurons) where a spike. Signal is emitted upon reaching a spike threshold. Further details is described in Section 1.3.

\section{Spiking Neural Networks}

The brain deals extremely well as a spatio-temporal information processing, where when presented with information, complex spatio-temporal paths and patterns are formed across the brain [4]. This has motivated researchers to create spatio-temporal data machine (STDM) for processing SSTD information based on the brain physiology. By imitating the behaviour of the brain analysing data as close as possible, a data processing model can be constructed - which can be later on used to process new upcoming stream of data.

Inspired by the brain biological mechanism, spiking neural networks has been introduced and proven to be more powerful than conventional neuron network models $[4,23]$. The idea is to apply a spiking neuron activation function on each individual neurons in a network - later on, used to measure the association strength between different SSTD components. A network of spiking neurons with spatial memory are capable of encoding, store, recognize, and recall spatial information patterns $[1,10]$, therefore offers potential to create a spatial memory model for analyzing spike patterns from a data stream.

Spiking neural networks have shown many possible implementations in various fields, as featured in Kasabov et al. [1, 4, 6]-[8, 11, 24]. For instance, a study [24] has been conducted using spiking neural network architecture to understand the functional changes in the brain for opiate dependent treatments. NeuCube EvoSpike architecture [4], has been developed to recognize brain signals pattern for integration with neuromorphic cognitive systems.

\section{METHODOLOGY}

Environmentally related data collected is first prepared into a format consisting variables and their corresponding measurements (in this case, the reading is acuired on daily basis). The Kuala Krai real-world reading includes measurements of daily rainfall $(\mathrm{mm})$, monthly rainfall $(\mathrm{mm})$, average daily temperature $\left({ }^{0} \mathrm{C}\right)$ wind speed $\left(\mathrm{ms}^{-1}\right)$, The last column of the measurement is labelled with classes, either 'High-Risk' or 'LowRisk', to be used for supervised learning.

Figure 1 visualises the spiking neural network architecture for analysing the environmental data. First, SSTD formatted data is encoded into input neurons which is then loaded into a three-dimensional data array acting as SNN reservoir. The environmental data is then trained using the network, where the SNN classifier later on classify the resulting data into 'High-Risk' and 'Low-Risk' class, determining the chances of flooding in a certain area. The network is continuously optimised and corrected using new loaded environmental data, and an optimised model is then saved upon achieving a satisfactory result. The created model can be continuously applied to further train and improve the accuracy of the network using new data.

The data in form of SSTD were encoded [11, 25] into input neurons, each represents an environmental-related feature extracted from the data source. Next, the features are stochastically transmitted among spatially distributed neurons (representing spatial component) on a three-dimensional network of spiking neurons-this creates a simple model of spiking neural network for analysing the input data. Each encoding process locates the input neurons to different positions, providing different chances of association between input neurons. 


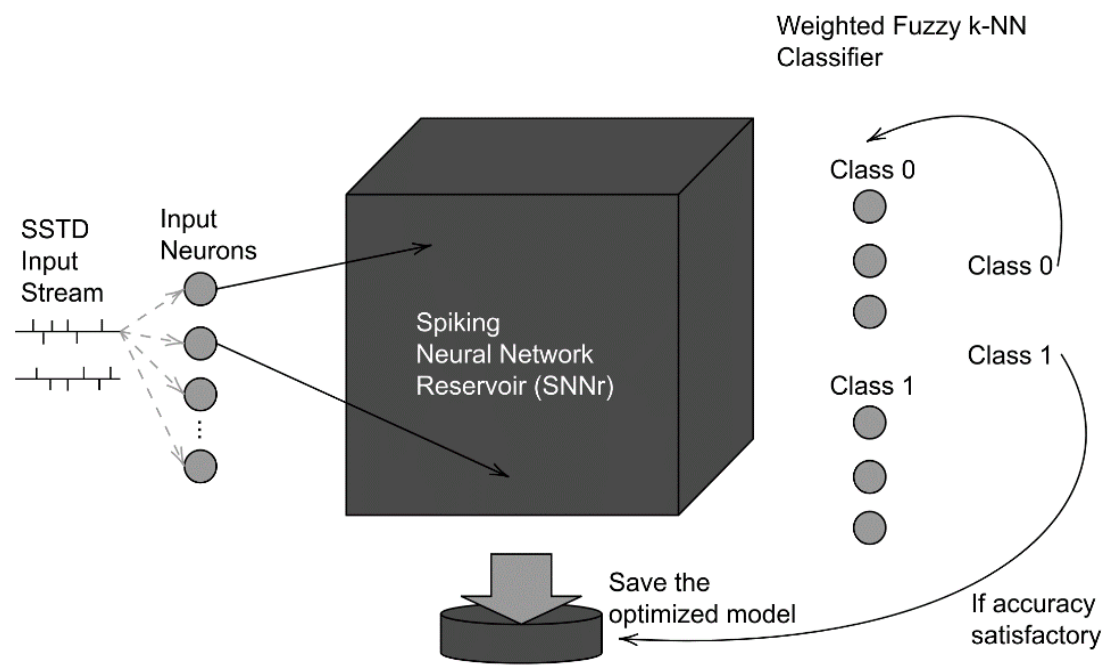

Figure 1. A spiking neural network architecture for capturing SSTD patterns

\subsection{Data Description}

Dataset used covers historical environmental data from 2012-2016 (5 years) provided by the Malaysia Meteorological Department. The first dimension is the temporal (time) dimension and the second dimesion is the reading of 6 features, measured once daily for 5 years based on Kuala Krai station; (i) water level in cm, (ii) daily rainfall in $\mathrm{mm}$, (iii) monthly rainfall in $\mathrm{mm}$, (iv) wind speed in $\mathrm{ms}^{-1}$, (v) air humidity in percentage, and (vi) temperature in degree Celcius. A "Class" label is pre-defined in an additional column for supervised learning mode and the value is determined by water level of a river cross-section as determined by Malaysian Metreological Department water level manual. Data is classified with the label 'High-Risk' if water level value surpasses a certain threshold value (determined in the manual), otherwise 'Low-Risk'.

Visualised in Figure 2, 'High-Risk' samples were created with 14-days time length; day-14 until day1 where measurement of the 6-features patterns gradually changes, approaching flood on day-0. Low-Risk samples were created by selecting a total of 14-days reading where there reading shows absoulute no flood, in which, to be used as a control class.

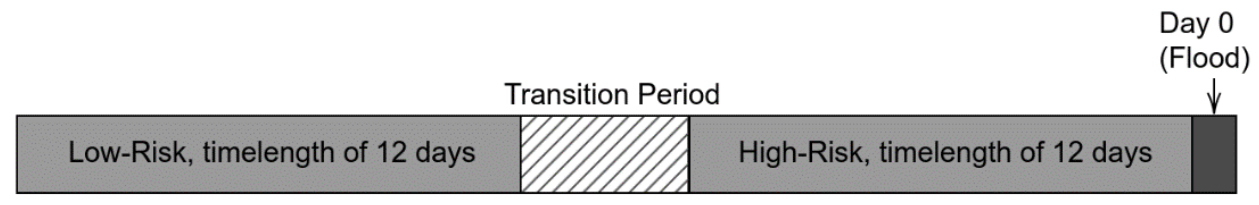

Day $12,11,10 \ldots 1$

Figure 2. Temporal data is fed backwards, from day-12, day-11, and so forth approaching flood day on day-0

Approaching to flood occurrence day, features measurement changes are expected in pattern such: water level increase, daily rainfall measurements increase, air humidity measurements increase; and temperature measurements decrease. Hidden patterns may be formed by monthly rainfall and wind speed reading. Spiking neural network is used to analyse and learn the hidden spike patterns from the environment data and reason about the variables compound which in total, can be used to estimate and label risk of flooding. The correlation between the features forms a model for processing and labelling the new incoming input data based on previousy trained data via supervised and unsupervised learning.

Over the five years, 7 flood occurrences have been observed, enabling 7 samples to be formed to train the network. Each sample consists of 'High-Risk' and 'Low-Risk' for a flood case study with a time length of 12-days for reading changes pattern observation.

\subsection{Experimental Procedures}

Experiments are conducted in such a way the data (pattern) model is first constructed, then simulated in two phases; (i) training and (ii) testing. The simulation has been conducted using several algorithms 
including multilayer perceptron (MLP) for Experiment 1, multiclass classification for experiment 2, and SNN architecture which encorporates wkNN classifier for experiment 3. Experiments were conducted by using the created samples and experiments were conducted to assess the risk of flood for 1-day earlier and 3-days earlier by using samples time length split as specified in Table 2 .

During experiments, the data in form of SSTD were encoded into input neurons, each represents an environmental-related feature extracted from the data source. Next, the features are stochastically transmitted among spatially distributed neurons (representing spatial component) on a three-dimensional network of spiking neurons-this creates a simple model of spiking neural network for analysing the input data. Each encoding process locates the input neurons to different positions, providing different chances of association between input neurons, in which, an optimal mapping of the input neurons in the spiking neural network cube creates the optimal model for validating the variables associations.

The output from the neural network cube is transmitted to a SNN classifier which classifies the result into 'High-Risk' and 'Low-Risk' for flooding based on the patterns constructed using previous days. The optimal model can be applied to new data for prediction of risk of flooding.

In order to achieve the best accuracy possible with less time and resource consumption, the experiments execution parameters have been optimized by using Grid Search Optimization algorithm, where set of limits have been introduced to the experiment execution parameters. Tuning to find the most suitable parameter without affecting the fair comparison between experimental execution has been prepared using configuration as presented in Table 3.

Table 2. Training and testing samples of different time length are used to feed the network with environmental data for an early prediction of flood risk

\begin{tabular}{cccc}
\hline Sample Type & $\begin{array}{c}\text { Data Split } \\
\text { Train:Test }\end{array}$ & $\begin{array}{c}\text { Percentage of } \\
\text { Trained Data/All (\%) }\end{array}$ & Early Day Predict \\
\hline 1 Day Early Sample & $12: 12$ & 100.00 & 1 \\
3 Days Early Sample & $10: 12$ & 83.30 & 3 \\
\hline
\end{tabular}

Table 3. Optimised parameters for experiment execution

\begin{tabular}{lccc}
\hline Parameter & Upper Limit Optimisation & Lower Limit Optimisation & Optimal Value \\
\hline AER Threshold & 1.0 & 0.1 & 0.5 \\
Small World Radius & 1.0 & 5.0 & 2.5 \\
STDP Rate & 0.01 & 0.10 & 0.01 \\
Firing Threshold & 0.1 & 1.0 & 0.47 \\
Refractory Time & 1 & 10 & 2 or 7 \\
Time Rounds & 1 & 10 & 4 or 5 \\
deSNN Mod & 0.01 & 0.50 & 0.157 \\
deSNN Drift & 0.01 & 0.50 & 0.329 \\
\hline
\end{tabular}

\section{RESULTS AND DISCUSSION}

The experiment has been executed using three different algorithms; experiment 1 has been executed using MLP algorithm, experiment 2 has been executed using Multiclass Classification algorithm, and experiment 3 has been executed using SNN with wkNN. The result is produced by comparing the correctness of the data classified by the network after undergoing supervised training and executed on unsupervised training based in Train:Test data split of 12:12 for 1-day earlier prediction and 10:12 for 3-days earlier prediction.

The result in Figure 3 shows that the overall accuracy of flood risk assessment produced by MLP, multiclass classifier, and SNN architecture wkNN. In general, the SNN with wkNN classifier has been trained to create a personalised model for assessing flood risk, while parameter optimisation over the model has assisted in producing the highest accuracy in assessing flood risk as compared to other algorithms.

In this respect, a more radical approach has been taken to (i) create a personalised data model and (ii) to create a data samples suitable to be used for generating the model for assessing the risk of flood. Most conventional global modelling techniques are able to classify result with high accuracy, but at the same time lacks the capability to consider temporal components into modelling the data model (for observing occurrences patterns and introduce the relativity between variables). Comparison between result achieved by conventional global modelling techniques with the SNN with wkNN classifier has demonstrated that personalised modelling performs better to produce a result with high accuracy by considering variables change patterns over temporal component. 


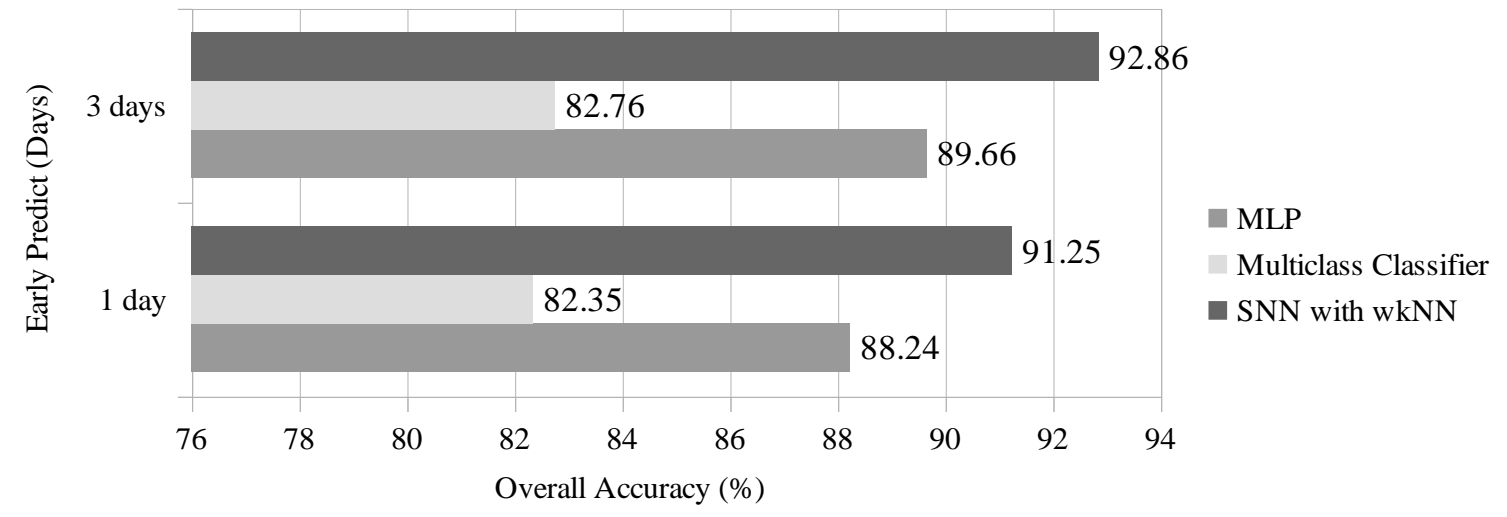

Figure 3. Result of flood case risk assessment for Kuala Krai for year 2012-2016 (5Y)

Noticeably, SNN with wkNN has produced a classification result with the highest accuracy as compared to MLP and multiclass classifier for both earlier 1-day and 3-days prediction of risk of flood. This simulation proves that personalised modelling is capable of producing a better classification result for a specific use case as compared to other data modelling techniques. It should be clarified that for flood case in Malaysia, early prediction is possible to be made up to 3-days early since flooding most commonly occurs due to continuous raining for few days. Observation in changes in environmental variables patterns using SNN has made the result possible.

\section{CONCLUSION}

The paper has presented an evolving Spiking Neural Networks methods for classification problems with a case study of real-world flood events risk assessment. The result of classification using SNN with wkNN has shown a significant increase in accuracy as compared to the conventional MLP and multiclass classifier due to the capability of the personalised model to represent a specific case study rather than the general (global) model. Personalised models can be created to represent each region of an area, where each region can be individually represented and analysed.

The outcome of the research may be applied in various ways including development of an alert system to predict environmental disaster such as flood occurrences. Development of such technology could help to reduce the risk of accidental death and property losses, by providing an earlier alert to be broadcast to a high risk area. In this case, the output value can be a classification value or a regression value where the output value is the reading of river water level. Another possible application of this research is to be integrated into a flood disaster management decision support system for property valuation in flood affected area; the system can analyse contributing factors in assessing the value of property depending on the risk prediction of the flood event.

\section{ACKNOWLEDGEMENTS}

The authors would like to acknowledge Universiti Tun Hussein Onn Malaysia (UTHM) and Ministry of Higher Education (MOHE) Malaysia for providing research facilities and fund via Fundamental Research Grant Scheme (FRGS) with project code: FRGS/1/2016/ICT02/UTHM/03/2.

\section{REFERENCES}

[1] N. Kasabov et al., "Evolving spatio-temporal data machines based on the NeuCube neuromorphic framework: Design methodology and selected applications," Neural Networks, vol. 78, no. December, pp. 1-14, 2015.

[2] N. Kasabov, "Global, local and personalised modeling and pattern discovery in bioinformatics: An integrated approach," Pattern Recognit. Lett., vol. 28, no. 6, pp. 673-685, 2007.

[3] N. Kasabov, "Soft computing methods for global, local and personalized modeling and applications in bioinformatics," Stud. Comput. Intell., vol. 196, pp. 1-18, 2009.

[4] N. Kasabov, "NeuCube EvoSpike architecture for spatio-temporal modelling and pattern recognition of brain signals," Lect. Notes Comput. Sci. (including Subser. Lect. Notes Artif. Intell. Lect. Notes Bioinformatics), vol. 7477 LNAI, pp. 225-243, 2012. 
[5] R. N. Hartono, R. Pears, N. Kasabov, and S. P. Worner, "Extracting temporal knowledge from time series: A case study in ecological data," Proc. Int. Jt. Conf. Neural Networks, pp. 4237-4243, 2014.

[6] M. Othman, "Stroke risk prediction using Spiking Neural Network method," Knowledge Engineering and Discovery Research Institute - AUT, 2015. [Online]. Available: https://kedri.aut.ac.nz/neucube/stroke. [Accessed: 19-Feb2019].

[7] E. Tu et al., "NeuCube(ST) for spatio-temporal data predictive modelling with a case study on ecological data," Proc. Int. Jt. Conf. Neural Networks, no. August 2015, pp. 638-645, 2014.

[8] N. Kasabov et al., "Evolving spiking neural networks for personalised modelling, classification and prediction of spatio-temporal patterns with a case study on stroke," Neurocomputing, vol. 134, pp. 269-279, 2014.

[9] M. Othman et al., "Improved predictive personalized modelling with the use of Spiking Neural Network system and a case study on stroke occurrences data," Proc. Int. Jt. Conf. Neural Networks, pp. 3197-3204, 2014.

[10] T. Madl, K. Chen, D. Montaldi, and R. Trappl, "Computational cognitive models of spatial memory in navigation space: A review," Neural Networks, vol. 65, pp. 18-43, 2015.

[11] N. K. Kasabov, "NeuCube: A spiking neural network architecture for mapping, learning and understanding of spatiotemporal brain data," Neural Networks, vol. 52, pp. 62-76, 2014.

[12] M. Othman, S. A. Mohamed, M. H. A. Abdullah, M. M. Yusof, and R. Mohamed, "A framework to cluster temporal data using personalised modelling approach,” Adv. Intell. Syst. Comput., vol. 700, pp. 181-190, 2018.

[13] Z. Li et al., "Spatio-temporal forecasting of weather-driven damage in a distribution system," IEEE Power Energy Soc. Gen. Meet., vol. 2015-Septe, 2015.

[14] M. H. A. Abdullah, H. Mahdin, M. F. M. Fudzee, M. A. Salamat, A. A. Ramli, and S. Kasim, "An improved computational framework using one stage filtration by incorporating knowledge in gene expression clustering," pp. $1-5,2016$.

[15] W.-D. Vogl et al., "Spatio-Temporal Signatures to Predict Retinal Disease Recurrence," Inf. Process. Med. Imaging, vol. 24, no. $15929,2015$.

[16] V. Lakshmanan, I. Adrianto, T. Smith, and G. Stumpf, "A spatiotemporal approach to tornado prediction," in Proceedings of the International Joint Conference on Neural Networks, 2005, vol. 3, pp. 1642-1647.

[17] [N. Kasabov, "Data analysis and Predictive Systems and Related Methodologies - Personalised Trait Modelling System.," New Zeal. Pat. No. 572036, PCT/NZ2009/000222, NZ2009/000222-W16-79, vol. 1, no. 19, 2008.

[18] A. Ab. Ghani, C. K. Chang, C. S. Leow, and N. A. Zakaria, "Sungai Pahang digital flood mapping: 2007 flood," Int. J. River Basin Manag., vol. 10, no. 2, pp. 139-148, 2012.

[19] P. Y. Julien, a. A. Ghani, N. a. Zakaria, R. Abdullah, and C. K. Chang, "Case Study: Flood Mitigation of the Muda River, Malaysia,” J. Hydraul. Eng., vol. 136, no. 4, pp. 251-261, 2010.

[20] S. K. Sinnakaudan, A. Ab Ghani, M. S. S. Ahmad, and N. A. Zakaria, "Flood risk mapping for Pari River incorporating sediment transport," Environ. Model. Softw., vol. 18, no. 2, pp. 119-130, 2003.

[21] S. Pang and N. Kasabov, "Inductive vs transductive inference, global vs local models: SVM, TSVM, and SVMT for gene expression classification problems," IEEE Int. Conf. Neural Networks - Conf. Proc., vol. 2, pp. 1197-1202, 2004.

[22] M. H. A. Abdullah, "Comparative Analysis of Spatio/Spectro-Temporal Data Modelling Techniques," in Data Engineering And Information Security, 1st ed., Penerbit UTHM, 2017.

[23] W. Maass, "Networks of spiking neurons: the third generation of neural network models," Neural Networks, vol. 10, no. 9, pp. 1659-1671, 1997.

[24] E. Capecci, N. Kasabov, and G. Y. Wang, "Analysis of connectivity in NeuCube spiking neural network models trained on EEG data for the understanding of functional changes in the brain: A case study on opiate dependence treatment," Neural Networks, vol. 68, pp. 62-77, 2015.

[25] E. Tu, N. Kasabov, and J. Yang, "Mapping Temporal Variables Into the NeuCube for Improved Pattern Recognition, Predictive Modeling, and Understanding of Stream Data," IEEE Trans. Neural Networks Learn. Syst., vol. 28, no. 6, pp. 1305-1317, 2017.

\section{BIOGRAPHIES OF AUTHORS}

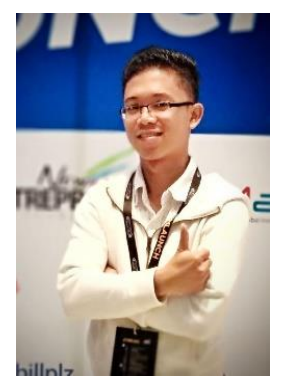

Mohd Hafizul Afifi Abdullah received his Bachelor in Computer Science (Web Technology) with Honors from Universiti Tun Hussein Onn Malaysia in 2017. He is currently a researcher assistant at Universiti Tun Hussein Onn Malaysia. Interested in data mining, pattern recognition, and outcome prediction. Specializes in spiking neural network and artificial intelligence.

ResearchGate: https://www.researchgate.net/profile/Mohd_Hafizul_Afifi_Abdullah 

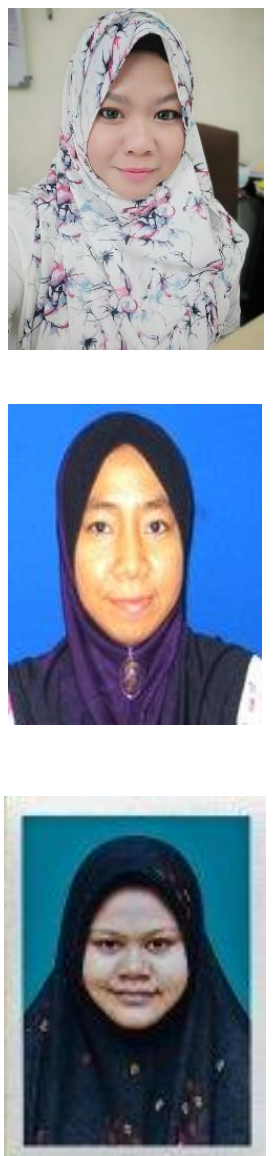

Muhaini Othman received her Bachelor in Information Technology from Northern University of Malaysia, Master in Computer Science from University of Malaya in 1999 and 2006 respectively and $\mathrm{PhD}$ in Computer and Mathematical Science from Auckland University of Technology in 2015. She is a Senior Lecturer at Faculty of Computer Science and Information Technology, Universiti Tun Hussein Onn Malaysia (UTHM). Her research interests include Personalised Predictive Modelling, Spiking Neural Networks and the representation of spatial and temporal data in ontology-based systems for personalised decision support. More information on Dr Muhaini can be found on FCSIT web site: http://fsktm.uthm.edu.my/

ResearchGate: https://www.researchgate.net/profile/Muhaini_Othman2

Shahreen Kasim is an Assistant Professor from the Department Web Technology, Universiti Tun Hussein Onn Malaysia Malaysia. The research interest includes soft computing and bioinformatics.

ResearchGate: https://www.researchgate.net/profile/Shahreen_Kasim

Siti Aisyah binti Mohamed is a graduate student of Bachelor in Information Technology from Universiti Tun Hussien Onn Malaysia (UTHM). She is currently pursuing master study by research at UTHM in which specializes at personalised modelling of temporal data in clustering approach. Further concerned topic is in the deep learning study on Spiking Neural Network of temporal data, features selection, and clustering approach.

ResearchGate: https://www.researchgate.net/profile/Siti_Mohamed15 